\title{
Feasibility of comprehensive genotyping specimens from radial endobronchial ultrasonography and electromagnetic navigation bronchoscopy
}

\author{
Maxime Robin ${ }^{1}$, Laurent Mhanna ${ }^{1}$, Leonor Chaltiel ${ }^{2}$, Gavin Plat $\mathbb{D}^{1}$, Valentin Héluain ${ }^{1}$, Céline Basset ${ }^{3,4}$, \\ Julie Meilleroux ${ }^{5}$, Thomas Filleron ${ }^{2}$, Julien Mazières ${ }^{1,4,6}$, Christophe Hermant $^{1}$ and Nicolas Guibert ${ }^{1,4,6}$
}

${ }^{1}$ Pulmonology Dept, Larrey University Hospital, Toulouse, France. ${ }^{2}$ Biostatistics Dept, Institut Claudius Regaud, Toulouse University Cancer Institute (IUCT-O), Toulouse, France. ${ }^{3}$ Cytology Dept, IUCT-O, Toulouse, France. ${ }^{4}$ Université de Toulouse III Paul Sabatier, Toulouse, France. ${ }^{5}$ Pathology Dept, IUCT-O, Toulouse, France. ${ }^{6}$ Cancer Research Centre of Toulouse (CRCT), Inserm, Toulouse, France.

Corresponding author: Nicolas Guibert (guibert.n@chu-toulouse.fr)

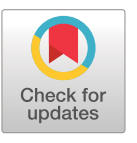

Copyright @The authors 2021

This version is distributed under the terms of the Creative Commons Attribution NonCommercial Licence 4.0. For commercial reproduction rights and permissions contact permissions@ersnet.org

Received: 15 Dec 2020 Accepted: 12 May 2021

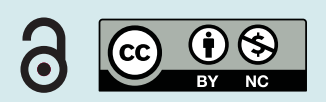

Shareable abstract (@ERSpublications)

Radial EBUS and EMN bronchoscopies are safe and sensitive procedures for lung cancer diagnosis. Cytology is highly complementary with histology. These small samples are, however, not suitable for an exhaustive molecular testing in $30 \%$ of cases. https://bit.ly/3eZ7Xn0

Cite this article as: Robin M, Mhanna L, Chaltiel L, et al. Feasibility of comprehensive genotyping specimens from radial endobronchial ultrasonography and electromagnetic navigation bronchoscopy. ERJ Open Res 2021; 7: 00942-2020 [DOI: 10.1183/23120541.00942-2020].

\section{Abstract}

Introduction Mini-invasive bronchoscopic techniques (such as radial endobronchial ultrasonography (rEBUS) and electromagnetic navigation (EMN)) have been developed to reach the peripheral lung but result in small samples. The feasibility of an adequate molecular testing from these specimens has been very little studied.

Methods We retrospectively reviewed EMN and rEBUS procedures performed in patients diagnosed with lung cancer in our institution in 2017 and 2018. We analysed the sensitivity for rEBUS and EMN and each sampling method, and the feasibility of a comprehensive molecular testing.

Results In total, 317 rEBUS and 14 EMN were performed. Median sizes of tumours were 16 and $32 \mathrm{~mm}$ for EMN and rEBUS, respectively. Overall sensitivity for rEBUS and EMN was 84.3\%. Cytology was found to be complementary with biopsies, with $13.3 \%$ of cancer diagnosed on cytology while biopsies were negative. Complication rate was $2.4 \%$ (pneumothorax $1.5 \%$, mild haemoptysis $0.9 \%$ ). Genotyping (immunohistochemistry for ROS1 and $A L K$ followed by fluorescence in situ hybridisation if positive and hybrid capture next-generation sequencing covering 48 genes), when ordered $(\mathrm{n}=188)$, was feasible in 69.1\% (EGFR 17.7\%, KRAS 31.7\%, BRAF 4.8\%, ALK 1.2\%, MET 3.1\%, HER2 0.8\%). PD-L1 (programmed death-ligand 1) expression, when ordered $(n=232)$, could be analysed in $94 \%$ of cases. Overall, 56.9\% (33 out of 58) of patients for whom genotyping was not feasible underwent a second sampling (12 pretreatment, 21 at progression), allowing for the detection of six actionable genotypes (five EGFR, one MET).

Conclusion rEBUS and EMN are sensitive and safe procedures that result in limited samples, often not suitable for genotyping, highlighting the importance of integrating liquid biopsy in routine testing.

\section{Introduction}

Mechanisms of oncogenesis in lung cancer have been largely deciphered over the past 20 years. Lung adenocarcinoma can now be considered as a cluster of discrete molecular subtypes, the majority being defined by a single alteration of an oncogenic driver. Multiplex genotyping and high-throughput genomic profiling by next-generation sequencing (NGS) is thus increasingly refining molecular diagnoses [1]. In addition, immune checkpoint inhibitors also require tissue for the analysis of the tumour micro-environment, in particular PD-L1 (programmed death-ligand 1) expression [2]. 
There is currently a paradox between the need to obtain significant amounts of sample to test an increasing number of biomarkers and the development of bronchoscopic minimally invasive techniques, resulting in small tissue samples with limited amounts of DNA [3]. Between 20 and $30 \%$ of EBUS-TBNA (endobronchial ultrasound transbronchial needle aspiration) nodes samples are rejected for genotyping because of lack of tissue [4, 5]. For peripheral lesions, bronchoscopy currently constitutes the preferred approach as it is less invasive than radio-guided biopsies [6]. The sensitivity of the main technologies, electromagnetic navigation (EMN) and radial EBUS (rEBUS), is 75\%, with very few complications $[6,7]$. The feasibility of adequate molecular testing of these specimens has, however, been less studied, in limited series [8] and without NGS [9].

In this study we aimed to use a large cohort to investigate: 1) the sensitivity of rEBUS and EMN, distinguishing cytology (brushings, washings) and histology (forceps biopsies) yields; 2) the feasibility of an exhaustive genotyping (including NGS) on these specimens, as well as of PD-L1 expression analysis; and 3 ) the impact of the latter on management of patients (rate and results of second biopsies).

\section{Methods}

\section{Patients}

We retrospectively studied consecutive patients who underwent as a first diagnostic procedure a bronchoscopy with rEBUS or EMN in 2017 and 2018 in the bronchoscopy unit of Toulouse University Hospital and were subsequently diagnosed with lung cancer, either by the procedure or later on. We reviewed data from all patients undergoing rEBUS or EMN in the Orbis ${ }^{\mathrm{TM}}$ Clinical Information System (AGFA Healthcare ${ }^{\mathrm{TM}}$ ), and the Occitanie oncology comprehensive database (http://www.onco-occitanie.fr). Patients gave their consent for this retrospective study and data were de-identified.

\section{Sampling}

EMN procedures (Superdimension system; Covidien, MA, USA) were performed under general anaesthesia through a laryngeal mask. This technology combines virtual navigation imaging with sensing of the position of a bronchoscopic catheter, matching virtual and real bronchial trees. Brushings and biopsies were performed through the guiding catheter after reaching the lesion.

Radial EBUS procedures were performed under local or general anaesthesia through a flexible bronchoscope. Dedicated brush and forceps were used through the 2.0-mm diameter GuideSheath (Olympus TM, Tokyo, Japan) to sample the lesions after detection of the lesion using the radial ultrasonic miniature probe (UM-S20-17S).

For both techniques, the guiding catheter was rinsed at the end of the procedure with saline for cytology.

\section{Sample handling: diagnosis and molecular testing}

Both cytology (brushing and catheter rinse) and histology (biopsies) samples were used for morphological diagnosis. Molecular testing was performed after DNA extraction from sections cut from cell (cytology) or formalin-fixed paraffin-embedded (tissue) blocks, and included a first screening of EGFR (epidermal growth factor receptor) common mutations by the Cobas ${ }^{\circledR}$ technique (Kit ROCHE Cobas DNA Sample Preparation Kit), immunohistochemistry (IHC) for ROS1 (Clone D4D6; Ozyme, Saint-Cyr-l’École, France) and ALK (anaplastic lymphoma kinase, Clone 1A4; Diagomics, Blagnac, France) rearrangements followed by fluorescence in situ hybridisation (FISH) if positive (IQFISH break-apart probe, Dako Omnis, Agilent, Santa Clara, CA, USA)), and in a second time, a hybrid capture NGS, covering a 48-genes panel (Roche Sequencing (Kapa/SeqCap), Roche, Bale, Switzerland; MiSeq DX, Illumina, San Diego, CA, USA). PD-L1 expression was assessed on biopsies and surgically resected specimens using IHC (Clone QR1, Quartett, Diagomics).

\section{Outcomes}

The primary outcome was the feasibility of an exhaustive molecular testing. Secondary outcomes were: 1) the overall sensitivity of the procedures and the sensitivities of cytology and histology specimens; 2) the number of patients undergoing second biopsy (before or after first-line treatment) and the molecular profile on these second samples; and 3) the feasibility of PD-L1 expression analysis and the concordance with tissue.

\section{Statistical analysis}

Data were summarised by frequency and percentage for categorical variables and by median and range for continuous variables. Comparisons between groups were done using chi-squared test or Fisher test for qualitative values. Comparison between biopsy and surgical specimens was done using the McNemar 
test for paired qualitative data. For all statistical tests, differences were considered significant at the 5\% level. All statistical analyses were conducted using STATA 16.1 software.

\section{Results}

Population

In total, 331 patients underwent rEBUS (n=317) or EMN ( $=14)$ procedures in 2017 and 2018. Median age was 68 years; $67.1 \%$ were male, $13.9 \%$ nonsmokers or light smokers ( $<10$ pack-years). The main characteristics of the population are reported in table 1.

Median size of the sampled lesion was $32 \mathrm{~mm}$ (min-max: 9-100) and was $\geqslant 30 \mathrm{~mm}$ for 205 patients (63.3\%). For EMN patients, median size was $16 \mathrm{~mm}$ (min-max: 10-30). The characteristics of the lesions are detailed in table 2 . Overall, $39.5 \%, 24.7 \%$ and $35.8 \%$ of patients had a metastatic, locally advanced (IIIA-IIIB) and localised lesion (I-IIB), respectively. Adenocarcinoma was the most frequent histology. Other subtypes are reported in figure $1 \mathrm{a}$.

\section{Diagnosis}

Overall sensitivity was $84.3 \%$ (279 out of 331); $85.4 \%$ (271 out of 317 ) for rEBUS and 57.1\% (8/14) for EMN. When performed, sensitivity of histology and cytology samples were $73.8 \%$ (234 out of 317) and $77.5 \%$ (234 out of 302), respectively (table 3). When both were performed, sensitivity was $86.7 \%$ (255 out of 294), and diagnosis was obtained on cytology only in 13.3\% (39 out of 294) and on histology only in $9.2 \%$ (27 out of 294$)$. When tumour size was available $(n=310)$, sensitivity was $89.4 \%$ (93 out of 107$)$ for lesions $<30 \mathrm{~mm}$ and $85.5 \%$ (171 out of 203 ) for lesions $\geqslant 30 \mathrm{~mm}$.

\section{Complications}

Eight complications (2.4\%) were reported, five (1.5\%) pneumothoraxes including one requiring chest tube insertion $(0.3 \%)$ and three $(0.9 \%)$ cases of mild haemoptysis, one of which required an additional day of hospitalisation.

\section{Feasibility of genotyping and PD-L1 expression analysis}

Tumour genotyping was ordered for 188 out of 331 patients (56.8\%, including 49 stage I-II, 30 stage IIIA and 108 stage IIIB-IV, 1 missing) and was not feasible in 30.9\% (58 out of 188) of cases due to exhausted tumour tissue (either no block left after the diagnostic steps, or insufficient DNA amount $(<5 \mathrm{ng})$ after extraction). Feasibility tended to be higher for advanced stages (72.5\%) compared to stage I-II (59.2\%, $\mathrm{p}=0.08$ ). Because tissue from biopsies was exhausted, genotyping was performed on cytology samples in $5.3 \%$ of cases. The main genotypes of interest identified are summarised in figure 1b: 17.7\% EGFR, 31.7\% KRAS (V-Ki-ras2 Kirsten rat sarcoma viral oncogene homologue), 4.3\% STK11 (serine/threonine kinase 11), 4.8\% BRAF, 3.1\% MET and 0.8\% HER2 (human epidermal growth factor receptor-2) mutations. ALK and ROS1 rearrangements could be tested using IHC for 167 out of 188 (88.8\%) patients (1.2\% ALK, confirmed by FISH, no ROS1).

PD-L1 expression analysis was ordered for 232 patients and was feasible in 94\% of cases (218 out of 232). Expression by at least $1 \%$ and $50 \%$ of tumour cells was detected in $49.5 \%$ and $22.7 \%$ of patients, respectively. Matched surgically resected pieces and tissue biopsies were available for 15 patients, showing

\begin{tabular}{|c|c|}
\hline Patients n & 331 \\
\hline Age, median (range) years & $68.0(42.0-87.0)$ \\
\hline$<70$ years & $177(53.5)$ \\
\hline$\geqslant 70$ years & $154(46.5)$ \\
\hline \multicolumn{2}{|l|}{ Sex } \\
\hline Male & $222(67.1)$ \\
\hline Female & 109 (32.9) \\
\hline \multicolumn{2}{|l|}{ Tobacco } \\
\hline Nonsmoker & $28(8.5)$ \\
\hline$<10$ pack-years & $18(5.4)$ \\
\hline 10-30 pack-years & $126(38.1)$ \\
\hline >30 pack-years & $159(48.0)$ \\
\hline
\end{tabular}




\begin{tabular}{|c|c|}
\hline \multicolumn{2}{|c|}{ Lesion size, median (range) mm } \\
\hline All & $32(9.0-100.0)$ \\
\hline rEBUS & $32(9.0-100.0)$ \\
\hline EMN & $16(10-30.0)$ \\
\hline Unknown, n (\%) & $46(16.1)$ \\
\hline \multicolumn{2}{|l|}{ Lesion size $(n=316)$} \\
\hline$<20 \mathrm{~mm}$ & $50(15.8)$ \\
\hline$\geqslant 20 \mathrm{~mm}$ & $266(84.2)$ \\
\hline Unknown, $\mathrm{n}$ & 15 \\
\hline \multicolumn{2}{|l|}{ Lesion size $(n=324)$} \\
\hline$<30 \mathrm{~mm}$ & $119(36.7)$ \\
\hline$\geqslant 30 \mathrm{~mm}$ & $205(63.3)$ \\
\hline Unknown, $\mathrm{n}$ & 7 \\
\hline \multicolumn{2}{|l|}{ Stage $(n=324)$} \\
\hline $\mathrm{I}-\mathrm{IIB}$ & $116(35.8)$ \\
\hline IIIA & $43(13.3)$ \\
\hline IIIB & $37(11.4)$ \\
\hline IV & $128(39.5)$ \\
\hline Unknown, n & 7 \\
\hline \multicolumn{2}{|l|}{ Histology $(n=331)$} \\
\hline Adenocarcinoma & $223(67.4)$ \\
\hline Squamous & $72(21.8)$ \\
\hline Small cell & $9(2.7)$ \\
\hline Carcinoid & $8(2.4)$ \\
\hline Undifferentiated & $6(1.8)$ \\
\hline Large cell & $4(1.2)$ \\
\hline Other & $4(1.2)$ \\
\hline Unknown & $5(1.5)$ \\
\hline
\end{tabular}

Data are presented as $\mathrm{n}(\%)$, unless otherwise stated. rEBUS: radial endobronchial ultrasonography; EMN: electromagnetic navigation.

a)

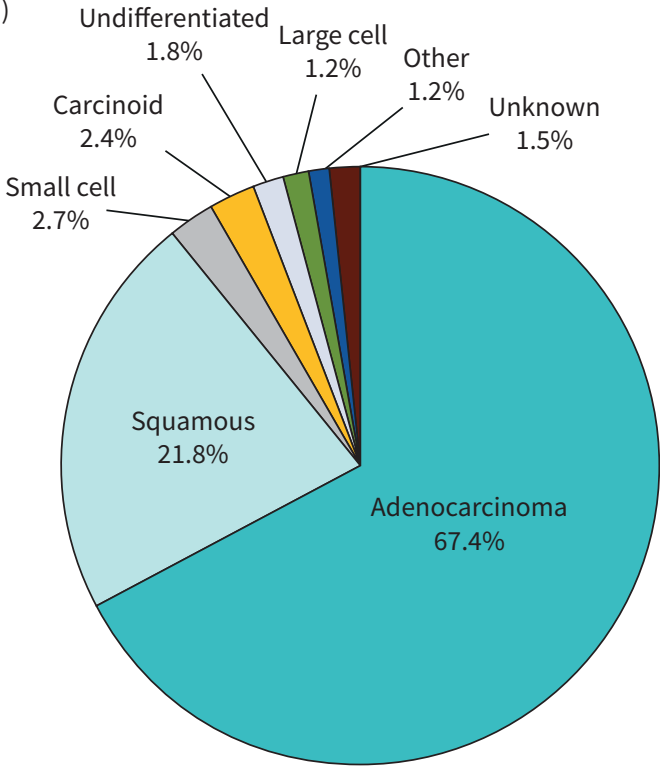

b)

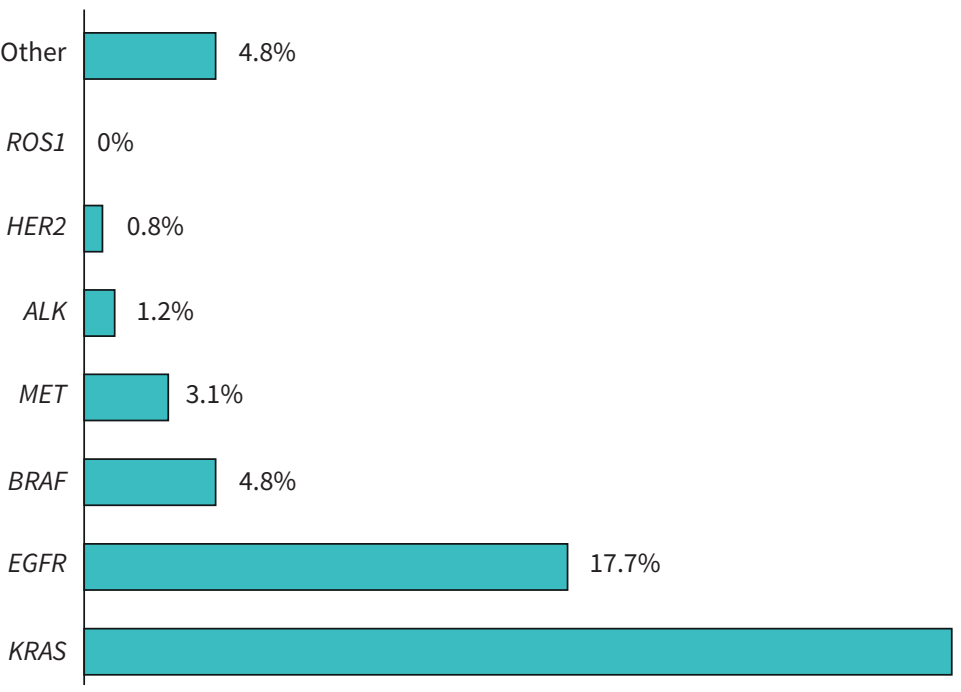

FIGURE 1 a) Histological subtypes and b) mutational status of the lung cancer diagnosed by radial endobronchial ultrasonography and electromagnetic navigation. 


\begin{tabular}{lc}
\hline TABLE 3 Diagnostic yield of bronchoscopy for peripheral lesions & \\
\hline Overall & $279 / 331(84.3)$ \\
rEBUS & $271 / 317(85.4)$ \\
\hline EMN & $8 / 14(57.1)$ \\
Cytology & $234 / 302(77.5)$ \\
\hline Brushings & $208 / 286(72.7)$ \\
\hline Rinse & $193 / 272(71)$ \\
\hline Histology & $234 / 317(73.8)$ \\
\hline Data are presented as n/N (\%). rEBUS: radial endobronchial ultrasonography; EMN: electromagnetic navigation. \\
\hline
\end{tabular}

a good concordance for the 50\% threshold (3 out of 3 tested positive and 12 out of 12 tested negative in both specimens). Of the eight patients tested $>1 \%$ on surgically resected specimens, three had tested negative on small biopsies.

\section{Second biopsies}

33 out of 58 (56.9\%) patients for whom genotyping was not possible underwent a second sampling, straight away $(\mathrm{n}=12)$ or at progression after first-line treatment $(\mathrm{n}=21)$. A screening plasma genotyping, limited to EGFR (Cobas), was proposed, completed with tissue when negative. The modalities and results of second biopsy procedures are detailed in the flow chart presented in figure 2. Overall, on plasma or second tissue biopsy genotyping, five additional EGFR (two in plasma, three on tissue), one non-V600E (pD594G) BRAF, one MET and five KRAS mutations were detected, before or after first-line treatment (figure 2).

\section{Discussion}

In this study, we extensively studied the performance of two bronchoscopy procedures (rEBUS and EMN) for peripheral lesions. We focused not only on the well-investigated diagnostic accuracy of these tools, but mostly on their limitations, in particular the pitfall of these scarce specimens in the era of personalised medicine.

First, we report an excellent sensitivity (84.3\%) of bronchoscopy for peripheral lesions and confirm the favourable safety profile [10] (1.5\% pneumothorax, only one requiring chest tube insertion (0.3\%)). These results appear slightly above what is usually reported $[6,7,11]$, likely in part linked to a high median size of the lesions (32 mm), the systematic use of the GuideSheath with rEBUS and the learning curve of a technique widely used in our institution since 2014. The sensitivity is higher in our experience for rEBUS (85.4\%) compared with EMN (57.1\%), a technology we only use for complex situations (twisted path to reach the lesion, ground glass nodules (less visible in ultrasonography [12]) and smaller nodules (median $16 \mathrm{~mm}$ compared to $32 \mathrm{~mm}$ for rEBUS in our cohort)).

An important point of our results is the complementarity between histology and cytology. Noteworthy, when both were done, $13.3 \%$ and $9.2 \%$ of diagnoses were obtained solely with cytology or histology alone, respectively, with a combined sensitivity of $86.7 \%$. We thus strongly suggest to systematically obtain cytology samples during rEBUS or EMN. The forceps are sometimes difficult to open correctly in distal airways. Brushing when biopsies are poorly productive can, in our experience, help open thin airway walls and increase the yield of rEBUS, in particular for eccentric lesions. This complementarity of cytology with forceps had already been suggested for transbronchial needle aspiration (TBNA) [13], a tool less used. TBNA procedures were performed in 16.5\% of cases in the AQuIRE registry, in part because the needle cannot always navigate sharp turns and was found to be very useful for eccentric lesions (diagnostic in 9.5\% when biopsies are negative) [14].

Another interesting point is the equivalent sensitivity for smaller lesions (89.4\% for lesions $<30 \mathrm{~mm}$ compared to $85.5 \%$ for lesions $\geqslant 30 \mathrm{~mm}$ ). This is an attractive result that should reinforce the place of these mini-invasive bronchoscopic diagnostic (and potentially therapeutic [15]) procedures for lung nodules, especially after the recent positive outcomes of the lung cancer screening NELSON trial [16].

But the main aim of our study was to investigate the ability of rEBUS and EMN to provide sufficient material for adequate molecular testing. A comprehensive genotyping is now required before treating patients with advanced nonsmall cell lung cancer, with an increasing number of available targeted 


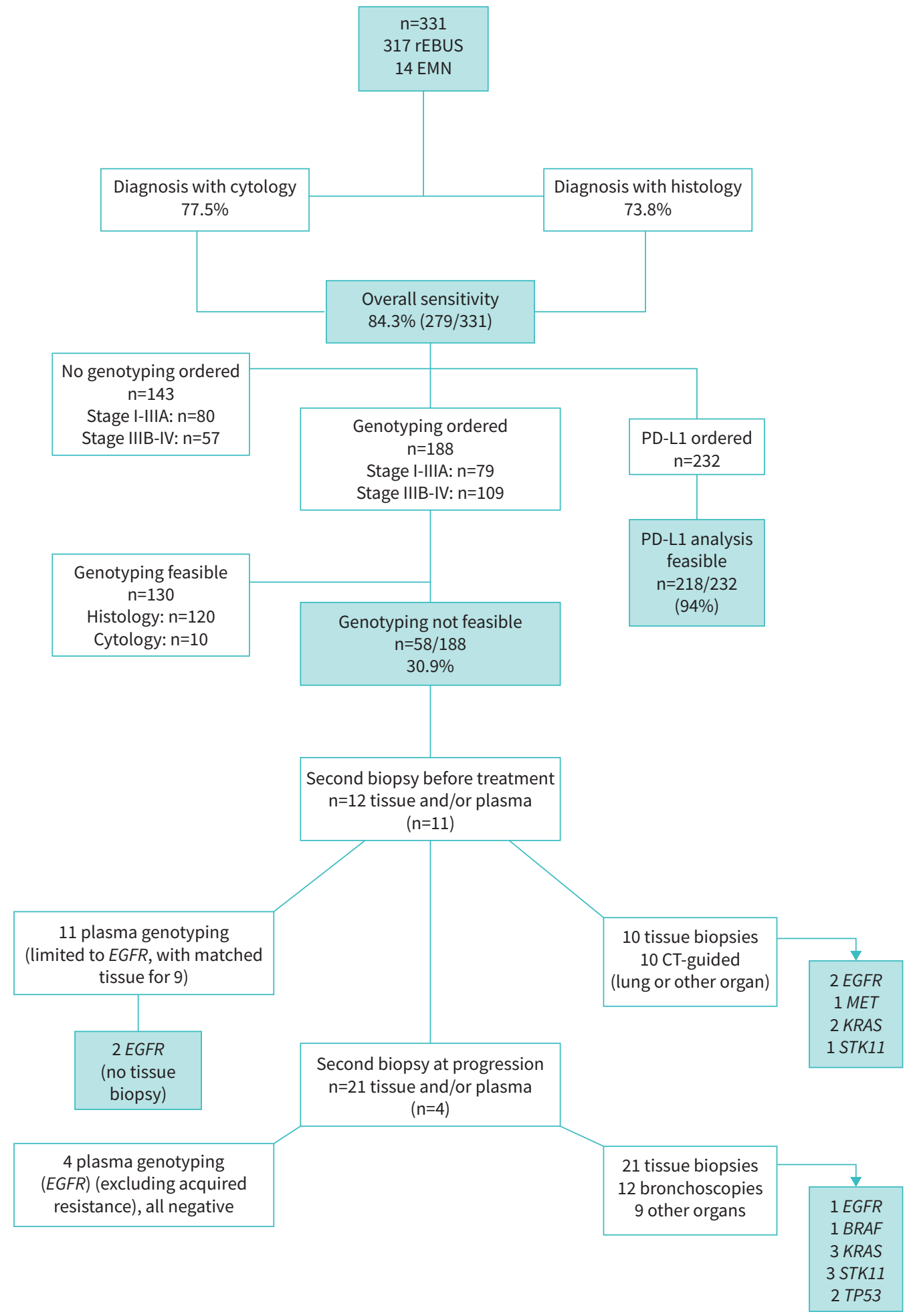

FIGURE 2 Flow chart of the study. rEBUS: radial endobronchial ultrasonography; EMN: electromagnetic navigation; CT: computed tomography; PD-L1: programmed death-ligand 1.

therapies, creating a paradox with the development of mini-invasive bronchoscopic procedures that results in limited material, often exhausted by the routine diagnostic steps. Up to $25 \%$ of patients receive treatment without knowledge of their mutational status [17]. 
In our study, 30.9\% of samples obtained by rEBUS or EMN were not suitable for an adequate molecular testing (sequential screening for the most prevalent genotypes followed with NGS) due to exhausted tissue after the diagnostic steps. Few studies have reported higher feasibilities using these specimens. GuisIER et al. [9] showed on a retrospective analysis of 111 patients that a multiplex analysis (without NGS) could be performed in $79 \%$ of rEBUS samples, cytology being more challenging. Others have showed excellent feasibilities for molecular testing but only focusing on one (EGFR [11, 18]) or few (EGFR, KRAS, BRAF [8]) specific genotypes. To our knowledge, this is the first study investigating the ability of these small specimens to provide a full molecular profile, including NGS, ALK and ROS1 rearrangements and PD-L1 expression.

We tried to investigate the impact of the limited feasibility of genotyping on patients' management (figure 2). 33 of the 58 patients for whom genotyping was not possible underwent a second sampling (12 before any treatment, 21 at progression), including 31 tissue invasive biopsies, i.e. 16.5\% of patients for whom a genotyping was ordered (31 out of 188). Two EGFR mutations were detected in blood, avoiding a tissue biopsy, and four additional "actionable" genotypes were found on second tissue biopsies (3 EGFR, 1 MET).

Obviously, these results only apply to the specific handling of our samples (DNA extraction from formalin-fixed specimens, hybrid capture NGS, MiSeq DX Illumina platform), and other approaches may result in a higher yield. There are several perspectives to compensate for the limited amount of tissue generated by mini-invasive sampling procedures in lung cancer: 1) liquid biopsy (which was limited in our centre in 2017 and 2018 to EGFR detection by Cobas in ctDNA) could only avoid two tissue biopsies in our study, but may represent a much more appealing approach in the future with the development of multiple circulating tumour DNA NGS platforms that cover all genotypes, including not only mutations but also amplifications or gene fusions with sensitivities ranging from $70 \%$ to $80 \%$ [19, 20]; 2) cryobiopsy, with the development of thin cryoprobes suited for peripheral lesions, could provide larger tissue specimens [21, 22]; 3) the use of non-formalin tissue fixation [23]; and 4) an alternative handling of cytology specimens, with in particular the use of the free-floating DNA present in their supernatant [24], may increase the overall yield of pauci-cellular biospecimens [25].

Finally, we have studied the feasibility of PD-L1 expression assessment on rEBUS and EMN samples, and confirmed it was highly feasible (94\%) [26]. We did not have enough matched surgically resected specimens to draw conclusions $(n=15)$, but $>50 \%$ of PD-L1 expressions detected on surgically resected specimens were correctly assessed on the small specimens, while three patients who tested negative had a $>1 \%$ expression on the surgical specimen, which tend to confirm that small samples can underestimate PD-L1 expression [27, 28].

In conclusion, this study confirms the good sensitivity of bronchoscopy with rEBUS and EMN for lung cancer diagnosis, even for small lesions $(<30 \mathrm{~mm})$, and its safety, and strongly highlights the complementarity of cytology with histology. However, it demonstrates that these small samples are not suitable for an exhaustive molecular testing in 30\% of cases, a significant issue given the multiplication of targetable genomic alterations. This pitfall could however be compensated by new techniques (rEBUS-guided cryobiopsy) providing larger samples, the use of cytology supernatant's free-floating DNA and, most of all, the implementation of plasma NGS that will in the near future limit the yield of second biopsy for genotyping.

Data availability: Data are available immediately after publication to researchers who provide a methodologically sound plan.

Conflict of interest: None declared.

References

1 Calvayrac O, Pradines A, Pons E, et al. Molecular biomarkers for lung adenocarcinoma. Eur Respir J 2017; 49: 1601734.

2 Reck M, Rodríguez-Abreu D, Robinson AG, et al. Pembrolizumab versus chemotherapy for PD-L1-positive non-small-cell lung cancer. N Engl J Med 2016; 375: 1823-1833.

3 Liam $\mathrm{C}-\mathrm{K}$, Mallawathantri S, Fong $\mathrm{KM}$. Is tissue still the issue in detecting molecular alterations in lung cancer? Respirology 2020; 25: 933-943.

4 Malapelle U, Bellevicine C, De Luca C, et al. EGFR mutations detected on cytology samples by a centralized laboratory reliably predict response to gefitinib in non-small cell lung carcinoma patients. Cancer Cytopathol 2013; 121: 552-560. 
5 Boulanger S, Delattre C, Descarpentries C, et al. Faisabilité de la recherche de mutations EGFR et KRAS sur des prélèvements obtenus par EBUS-PTBA [Feasibility of assessing EGFR mutation and others using samples obtained by EBUS transbronchial needle aspiration]. Rev Mal Respir 2013; 30: 351-356.

6 Steinfort DP, Vincent J, Heinze S, et al. Comparative effectiveness of radial probe endobronchial ultrasound versus CT-guided needle biopsy for evaluation of peripheral pulmonary lesions: a randomized pragmatic trial. Respir Med 2011; 105: 1704-1711.

7 Han Y, Kim HJ, Kong KA, et al. Diagnosis of small pulmonary lesions by transbronchial lung biopsy with radial endobronchial ultrasound and virtual bronchoscopic navigation versus CT-guided transthoracic needle biopsy: a systematic review and meta-analysis. PLoS One 2018; 13: e0191590.

8 Bonney A, Christie M, Beaty A, et al. The feasibility of molecular testing on cell blocks created from brush tip washings in the assessment of peripheral lung lesions. J Thorac Dis 2016; 8: 2551-2555.

9 Guisier F, Salaün M, Lachkar S, et al. Molecular analysis of peripheral non-squamous non-small cell lung cancer sampled by radial EBUS. Respirology 2016; 21: 718-726.

10 Ali MS, Trick W, Mba Bl, et al. Radial endobronchial ultrasound for the diagnosis of peripheral pulmonary lesions: a systematic review and meta-analysis. Respirology 2017; 22: 443-453.

11 Moon SM, Choe J, Jeong BH, et al. Diagnostic performance of radial probe endobronchial ultrasound without a guide-sheath and the feasibility of molecular analysis. Tuberc Respir Dis 2019; 82: 319-327.

12 Ikezawa $\mathrm{Y}$, Shinagawa N, Sukoh N, et al. Usefulness of endobronchial ultrasonography with a guide sheath and virtual bronchoscopic navigation for ground-glass opacity lesions. Ann Thorac Surg 2017; 103: 470-475.

13 Chao T-Y, Chien M-T, Lie C-H, et al. Endobronchial ultrasonography-guided transbronchial needle aspiration increases the diagnostic yield of peripheral pulmonary lesions: a randomized trial. Chest 2009; 136: 229-236.

14 Ost DE, Ernst A, Lei X, et al. Diagnostic yield and complications of bronchoscopy for peripheral lung lesions. Results of the AQuIRE registry. Am J Respir Crit Care Med 2016; 193: 68-77.

15 Steinfort DP, Herth FJF. Bronchoscopic treatments for early-stage peripheral lung cancer: are we ready for prime time? Respirology 2020; 25: 944-952.

16 de Koning $\mathrm{HJ}$, van der Aalst CM, de Jong PA, et al. Reduced lung-cancer mortality with volume CT screening in a randomized trial. N Engl J Med 2020; 382: 503-513.

17 Spicer J, Tischer B, Peters M. EGFR mutation testing and oncologist treatment choice in advanced NSCLC: global trends and differences. Ann Oncol 2015; 26: Suppl. 1, i57.

18 Yu K-L, Tsai T-H, Ho C-C, et al. The value of radial endobronchial ultrasound-guided bronchial brushing in peripheral non-squamous non-small cell lung cancer. Sci Rep 2018; 8: 5837.

19 Guibert N, Hu Y, Feeney N, et al. Amplicon-based next-generation sequencing of plasma cell-free DNA for detection of driver and resistance mutations in advanced non-small cell lung cancer. Ann Oncol 2018; 29: 1049-1055.

20 Guibert N, Pradines A, Favre G, et al. Current and future applications of liquid biopsy in non-small cell lung cancer from early to advanced stages. Eur Respir Rev 2020; 29: 190052.

21 Kho SS, Chan SK, Yong MC, et al. Performance of transbronchial cryobiopsy in eccentrically and adjacently orientated radial endobronchial ultrasound lesions. ERJ Open Res 2019; 5: 00135-2019.

22 Herath S, Yap E. Novel hybrid cryo-radial method: an emerging alternative to CT-guided biopsy in suspected lung cancer. A prospective case series and description of technique. Respirol Case Rep 2018; 6: e00287.

23 Gillespie JW, Best CJ, Bichsel VE, et al. Evaluation of non-formalin tissue fixation for molecular profiling studies. Am J Pathol 2002; 160: 449-457.

24 Durin L, Pradines A, Basset C, et al. Liquid biopsy of non-plasma body fluids in non-small cell lung cancer: look closer to the tumor! Cells 2020; 9: 2486.

25 Guibert N, Tsukada H, Hwang DH, et al. Liquid biopsy of fine-needle aspiration supernatant for lung cancer genotyping. Lung Cancer 2018; 122: 72-75.

26 Tsunoda A, Morikawa K, Inoue T, et al. A prospective observational study to assess PD-L1 expression in small biopsy samples for non-small-cell lung cancer. BMC Cancer 2019; 19: 546.

27 Sakata KK, Midthun DE, Mullon JJ, et al. Comparison of programmed death Ligand-1 immunohistochemical staining between endobronchial ultrasound transbronchial needle aspiration and resected lung cancer specimens. Chest 2018; 154: 827-837.

28 Ilie M, Long-Mira E, Bence C, et al. Comparative study of the PD-L1 status between surgically resected specimens and matched biopsies of NSCLC patients reveal major discordances: a potential issue for anti-PD-L1 therapeutic strategies. Ann Oncol 2016; 27: 147-153. 